\title{
Evaluation of the ACS NSQIP Surgical Risk Calculator in Elderly Patients Undergoing Hepatectomy for Hepatocellular Carcinoma
}

\author{
Kota Sahara $^{1,2}$. Anghela Z. Paredes ${ }^{1} \cdot$ Katiuscha Merath $^{1} \cdot$ Diamantis I. Tsilimigras $^{1} \cdot$ Fabio Bagante $^{3}$. \\ Francesca Ratti ${ }^{4}$. Hugo P. Marques ${ }^{5}$. Olivier Soubrane ${ }^{6}$. Eliza W. Beal ${ }^{1}$ • Vincent Lam ${ }^{7}$. George A. Poultsides ${ }^{8}$. \\ Irinel Popescu ${ }^{9} \cdot$ Sorin Alexandrescu ${ }^{9} \cdot$ Guillaume Martel $^{10} \cdot$ Workneh Aklile $^{10} \cdot$ Alfredo Guglielmi $^{3} \cdot$ Tom Hugh $^{11}$. \\ Luca Aldrighetti $^{4} \cdot$ Itaru Endo $^{2} \cdot$ Timothy M. Pawlik $^{1}$ (iD
}

Received: 8 January 2019 / Accepted: 20 February 2019 / Published online: 1 April 2019

(C) 2019 The Society for Surgery of the Alimentary Tract

\begin{abstract}
Background The American College of Surgeons National Surgical Quality Improvement Program (ACS NSQIP) surgical risk calculator (SRC) aims to help predict patient-specific risk for morbidity and mortality. The performance of the SRC among an elderly population undergoing curative-intent hepatectomy for hepatocellular carcinoma (HCC) remains unknown.

Methods Patients $\geq 70$ years of age who underwent hepatectomy for HCC between 1998 and 2017 were identified using a multiinstitutional international database. To estimate the performance of SRC, 12 observed postoperative outcomes were compared with median SRC-predicted risk, and C-statistics and Brier scores were calculated.

Results Among 500 patients, median age was 75 years (IQR 72-78). Most patients $(n=324,64.8 \%)$ underwent a minor hepatectomy, while $35.2 \%$ underwent a major hepatectomy. The observed incidence of venous thromboembolism (VTE) $(3.2 \%)$ and renal failure (RF) (4.4\%) exceeded the median predicted risk (VTE, 1.8\%; IQR 1.5-3.1 and RF, $1.0 \%$; IQR 0.52.0). In contrast, the observed incidence of 30-day readmission (7.0\%) and non-home discharge (2.5\%) was lower than medianpredicted risk (30-day readmission, 9.4\%; IQR 7.4-12.8 and non-home discharge, 5.7\%; IQR 3.3-11.7). Only 57.8\% and 71.2\% of patients who experienced readmission (C-statistic, 0.578 ; 95\% CI $0.468-0.688$ ) or mortality (C-statistic, 0.712 ; 95\%CI 0.5080.917 ) were correctly identified by the model.

Conclusion Among elderly patients undergoing hepatectomy for $\mathrm{HCC}$, the SRC underestimated the risk of complications such as VTE and RF, while being no better than chance in estimating the risk of readmission. The ACS SRC has limited clinical applicability in estimating perioperative risk among elderly patients being considered for hepatic resection of HCC.
\end{abstract}

Keywords NSQIP · Risk calculator · Elderly $\cdot$ Hepatectomy

\section{Introduction}

While representing only about $5 \%$ of all new cancer diagnoses worldwide, hepatocellular carcinoma (HCC) is the third

Timothy M. Pawlik

tim.pawlik@osumc.edu

1 Department of Surgery, The Urban Meyer III and Shelley Meyer Chair for Cancer Research, The Ohio State University Wexner Medical Center, 395 W. 12th Ave., Suite 670, Columbus, OH, USA

2 Department of Gastroenterological Surgery, Yokohama City University School of Medicine, Yokohama, Japan

3 Department of Surgery, University of Verona, Verona, Italy

4 Department of Surgery, Ospedale San Raffaele, Milan, Italy

5 Department of Surgery, Curry Cabral Hospital, Lisbon, Portugal leading cause of cancer-related mortality. ${ }^{1}$ In the USA, the mortality rate associated with liver cancer has increased over $30 \%$ since 2002 and the highest mortality rate has been reported among the elderly. ${ }^{2-4}$ An increasing life expectancy among

6 Department of Hepatobiliopancreatic Surgery, APHP, Beaujon Hospital, Clichy, France

7 Department of Surgery, Westmead Hospital, Sydney, Australia

8 Department of Surgery, Stanford University, Stanford, CA, USA

9 Department of Surgery, Fundeni Clinical Institute, Bucharest, Romania

10 Department of Surgery, University of Ottawa, Ottawa, Canada

11 Department of Surgery, The University of Sydney, School of Medicine, Sydney, Australia 
the general population has resulted in a growing number of elderly patients diagnosed with hepatic malignancies. ${ }^{5}$ For many patients, hepatic resection remains the best chance for cure. ${ }^{6}$ In light of medical advances and improvement in surgical technique, surgical patient selection has expanded and a greater number of complex patients-including older individuals with a higher comorbidity burden - are offered surgical resection. ${ }^{7}$ Thus, as the population continues to age, the proportion of elderly patients being considered for surgical intervention of a liver malignancy is expected to increase.

Concern exists, however, regarding the safety of hepatic resection among older patients. Indeed, elderly patients have more comorbidities and age-associated changes that can contribute to postoperative morbidity and mortality after major abdominal surgery. ${ }^{8-10}$ In fact, the rates of postoperative serious complications and mortality among older patients undergoing hepatopancreatobiliary (HPB) surgery have been reported to be as high as $50 \%$ and $6 \%$, respectively. ${ }^{11-15}$ Moreover, the development of severe postoperative complications may lead to a delay in return to home and receipt of adjuvant therapies, which can result in worse overall survival. ${ }^{16,17}$ As such, a thorough preoperative assessment of morbidity and mortality risk is critical when counseling individuals, especially among elderly patients.

In that context, the American College of Surgeons (ACS) developed the National Surgical Quality Improvement Program (NSQIP) online surgical risk calculator (SRC) based on data from over 3.8 million operations performed in 740 participating hospitals from 2012 to 2016. While risk calculators have been reported to be useful in predicting the risk of myocardial infarction or coronary death, surgical prognostication has been challenging due to the differences between the initial cohorts used to develop the tool and the "real-world" population. ${ }^{18,19}$ In fact, previous studies have reported a discrepancy between predicted risk by the SRC and observed outcomes among patients undergoing abdominal surgery. ${ }^{20,21}$ The performance of the ACS NSQIP SRC to predict outcomes among elderly patients undergoing hepatectomy for $\mathrm{HCC}$ has, however, not been assessed. As such, the objective of the current study was to determine the accuracy of the ACS NSQIP SRC to predict outcomes among elderly patients undergoing curative-intent liver resection for HCC using an international multi-institutional database.

\section{Methods}

\section{Study Population and Data Collection}

Patients 70 years of age or older who underwent curativeintent hepatectomy for histologically proven HCC with between 1998 and 2017 were identified using an international multi-institutional database (Fig. 1). Patients were enrolled from one of 11 major institutions, including The Ohio State University Wexner Medical Center, Columbus, OH, USA; Yokohama City University School of Medicine, Yokohama, Japan; University of Verona, Verona, Italy; Ospedale San Raffaele, Milano, Italy; Curry Cabral Hospital, Lisbon, Portugal; APHP, Beaujon Hospital, Clichy, France; Westmead Hospital, Sydney, Australia; Stanford University, Stanford, CA, USA; Fundeni Clinical Institute, Bucharest, Romania; University of Ottawa, Ottawa, Canada; and The University of Sydney, School of Medicine, Sydney, Australia. Hepatectomy was identified using current procedural terminology (CPT) codes $(47,120$, partial hepatectomy; 47,125, left hepatectomy; 47,130, right hepatectomy; 47,122 , trisegmentectomy). Partial hepatectomy was categorized as a minor hepatectomy, while left hepatectomy, right hepatectomy, and trisegmentectomy were categorized as a major hepatectomy. Patients who were less than 70 years of age, who did not have curative-intent surgery, or had ablation only were excluded. The study was approved by the Institutional Review Boards of all participating institutions.

The ACS NSQIP calculator was accessed online at http:// riskcalculator.facs.org/ on November 29 and 30, 2018. Individual patient data and respective CPT codes were entered into the calculator. Using the ACS NSQIP calculator, the incidence risk for the following 12 outcomes were estimated: serious complication, any complication, pneumonia, cardiac complication, surgical site infection (SSI), urinary tract infection (UTI), venous thromboembolism (VTE), renal failure, 30-day readmission, reoperation, 30-day mortality, and non-home discharge. Patient data required for calculation included procedure type, age group, sex, functional status, emergency case, American Society of Anesthesiologists (ASA) class, steroid use for chronic condition, ascites within 30 days prior to surgery, systemic sepsis within $48 \mathrm{~h}$ prior to surgery, ventilator dependence, disseminated cancer, diabetes mellitus, hypertension requiring medication, congestive heart failure 30 days prior to surgery, dyspnea, current smoker within 1 year, history of severe chronic obstructive pulmonary disease (COPD), dialysis, acute renal failure, height, and weight. For patients with a preoperative risk calculator factor missing, the risk was assumed to be at the lowest risk level. The "Surgeon Adjustment" was set at "1 -No adjustment necessary" for all cases.

\section{Data Analysis}

Categorical variables were presented as frequencies and percentages; continuous variables were presented as medians and interquartile ranges (IQR). The observed incidence of the varied outcomes was compared among patients undergoing minor and major hepatectomy. In addition, the proportions of observed outcomes were compared with the median- 
Fig. 1 Analytical sample of the patients included from the multiinstitutional international database

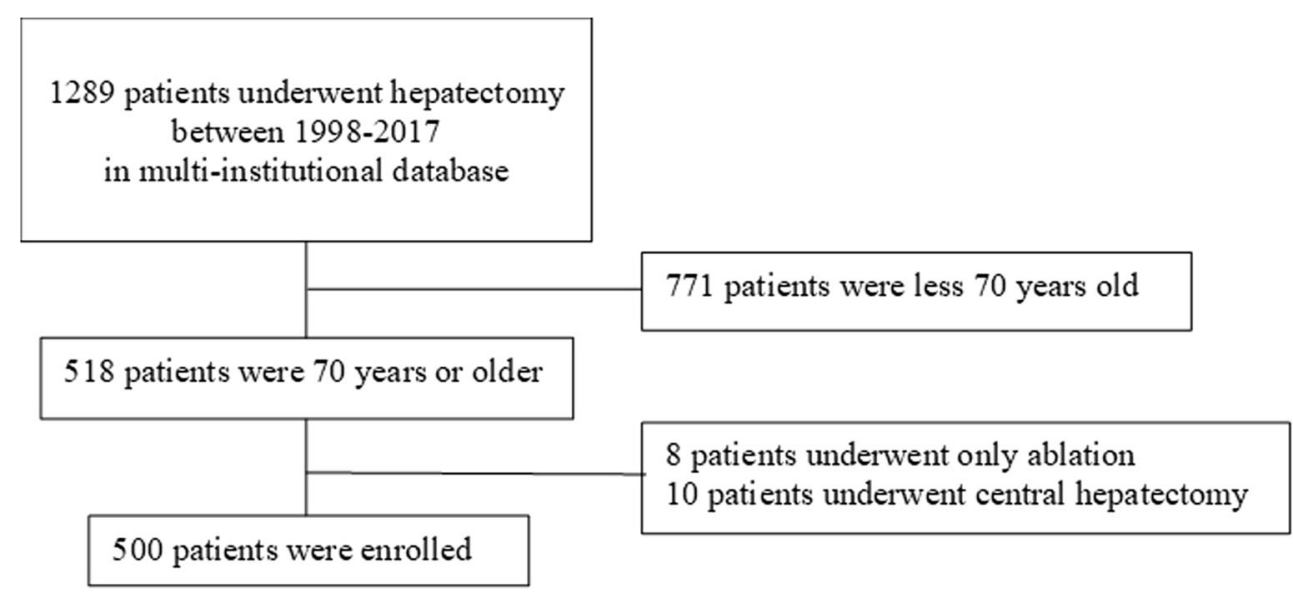

predicted risk according to the SRC. Concordance (C) statistic and Brier score were evaluated to assess the predictive ability of the SRC. The C-statistic represents the probability that an individual with the outcome of interest will have a higher predicted probability based on the logistic regression model compared with an individual who did not have the outcome. $^{22,23}$ A C-statistic of 0.5 indicates random concordance, while a value of 1.0 indicates a perfect predictive ability of the logistic regression model..$^{22}$ The Brier score measures the accuracy of probabilistic predictions; a score of 0 is associated with perfect predictive accuracy. ${ }^{24}$ Categorical variables were compared using chi-square tests and Fisher exact tests where appropriate. Continuous variables were compared using the Wilcoxon rank-sum tests and Kruskal-Wallis oneway analysis of variance. Logistic regression was utilized to examine the correlation between observed outcomes and the chance of outcome. Statistical significance was assessed at $\alpha=0.05$. Analyses of Brier score were performed using SAS v9.4. All other analyses were performed using SPSS, version 25 (IBM Corp).

\section{Results}

\section{Patient Characteristics and Postoperative Outcomes}

A total of 500 patients who underwent curative-intent hepatectomy for HCC were included in the analytic cohort (Table 1). Overall, median age was 75 years old (IQR 72$78)$ and the majority of patients were male $(n=367,73.4 \%)$. Roughly half of the patients were white $(n=229,45.8 \%)$ and the majority had an independent functional status $(n=414$, $82.8 \%$ ). The median Charlson comorbidity score index was 5 (IQR 2-6), while nearly half the patients were ASA class 2 $(n=237,47.4 \%)$. Roughly one in three patients had cirrhosis $(n=182,37.6 \%)$. Most patients $(n=324,64.8 \%)$ underwent a minor hepatectomy, while roughly one-third of patients $(n=$ $176,35.2 \%$ ) underwent a major hepatic resection (left hepatectomy $n=49,9.8 \%$; right hepatectomy $n=90,18 \%$; and trisegmentectomy $n=37,7.4 \%$ ). The vast majority of patients had an elective operation $(n=460,92 \%)$.

Following surgical resection (Table 2), roughly one in five patients $(21.4 \%)$ experienced a complication (minor hepatectomy $n=50,15.4 \%$ vs. major hepatectomy $n=57,32.4 \%$; $p<0.001)$. Overall, the most common postoperative complications were SSI $(n=37,7.5 \%)$, renal failure $(n=22,4.4 \%)$, and pneumonia ( $n=17,3.4 \%)$. Perhaps not surprising, compared with individuals who underwent a minor hepatectomy, a greater proportion of patients who underwent a major hepatectomy experienced a serious complication $(14.2 \%$ vs. $29.5 \% ; p<0.001)$. Similarly, the incidence of 30 -day readmission $(4.6 \%$ vs. $11.2 \% ; p=0.013)$ and reoperation $(0.9 \%$ vs. $4.6 \% ; p=0.006$ ) was higher among patients who underwent a major hepatic resection compared with patients who underwent a minor hepatic resection. Of note, the incidence of 30-day mortality $(0.9 \%$ vs. $2.3 \% ; p=0.250)$ and non-home discharge $(1.4 \%$ vs. $4.4 \% ; p=0.062)$ was similar among patients who underwent a minor versus major hepatectomy.

\section{Surgical Risk Calculator Outcome}

Table 3 summarizes the observed-event occurrence (percentage) versus the median-predicted risk (median percent predicted risk, IQR) among all patients for the various postoperative outcomes of interest. Of note, the observed incidence of VTE (3.2\%) and renal failure (4.4\%) exceeded the median-predicted risk for these outcomes (VTE, 1.8\%; IQR 1.5-3.1 and renal failure, 1.0\%; IQR 0.5-2.0). In contrast, the observed incidence of 30-day readmission (7.0\%) and nonhome discharge $(2.5 \%)$ was lower than the median-predicted risk (30-day readmission, 9.4\%; IQR 7.4-12.8 and non-home discharge, 5.7\%; IQR 3.3-11.7) (Table 3).

The NSQIP risk calculator was applied to all patients and the $\mathrm{C}$-statistic and Brier scores were calculated for all 12 surgical outcomes (Table 4). Patients who experienced a serious or any complication had a higher risk score approximately 
Table 1 Demographics and patient characteristics

\begin{tabular}{|c|c|}
\hline Variable & Total, $n(\%)$ \\
\hline Age - median (IQR) & $75(72,78)$ \\
\hline Male & $367(73.4)$ \\
\hline \multicolumn{2}{|l|}{ Race } \\
\hline White & $229(45.8)$ \\
\hline $\mathrm{AA}$ & $13(2.6)$ \\
\hline Asian & $184(36.8)$ \\
\hline Hispanic & $16(3.2)$ \\
\hline Other/unknown & $58(11.6)$ \\
\hline Charlson comorbidity score index — median (IQR) & $5(2,6)$ \\
\hline \multicolumn{2}{|l|}{ Functional status } \\
\hline Independent & $414(82.8)$ \\
\hline Partially dependent & $47(9.4)$ \\
\hline Dependent & $5(1.0)$ \\
\hline Missing & $34(6.8)$ \\
\hline \multicolumn{2}{|l|}{ ASA class } \\
\hline 1 & $30(6.0)$ \\
\hline 2 & $237(47.4)$ \\
\hline 3 & $165(33.0)$ \\
\hline 4 & $11(2.2)$ \\
\hline Missing & $57(11.4)$ \\
\hline \multicolumn{2}{|l|}{ Procedure type } \\
\hline Partial hepatectomy & $324(64.8)$ \\
\hline Left hepatectomy & $49(9.8)$ \\
\hline Right hepatectomy & $90(18.0)$ \\
\hline Trisegmentectomy & $37(7.4)$ \\
\hline \multicolumn{2}{|l|}{ Emergency surgery } \\
\hline Yes & $8(1.6)$ \\
\hline No & $460(92.0)$ \\
\hline Missing & $32(6.4)$ \\
\hline Height—median (IQR) & $166(159-172)$ \\
\hline Weight—median (IQR) & $69(59-80)$ \\
\hline \multicolumn{2}{|l|}{ Steroid use for chronic condition } \\
\hline Yes & $3(0.6)$ \\
\hline No & $439(87.8)$ \\
\hline Missing & $58(11.6)$ \\
\hline \multicolumn{2}{|l|}{ Ascites within 30 days prior to surgery } \\
\hline Yes & $15(3.0)$ \\
\hline No & $454(90.8)$ \\
\hline Missing & $31(6.2)$ \\
\hline \multicolumn{2}{|l|}{ Systematic sepsis within $48 \mathrm{~h}$ prior to surgery } \\
\hline Yes & $1(0.2)$ \\
\hline No & $468(93.6)$ \\
\hline Missing & $31(6.2)$ \\
\hline \multicolumn{2}{|l|}{ Ventilator dependent } \\
\hline Yes & 0 \\
\hline No & $479(95.8)$ \\
\hline Missing & $21(4.2)$ \\
\hline \multicolumn{2}{|l|}{ Disseminated cancer } \\
\hline Yes & $2(0.4)$ \\
\hline No & $476(95.2)$ \\
\hline Missing & $22(4.4)$ \\
\hline \multicolumn{2}{|l|}{ Diabetes } \\
\hline Yes & $203(40.6)$ \\
\hline No & $286(57.2)$ \\
\hline Missing & $11(2.2)$ \\
\hline \multicolumn{2}{|l|}{ Hypertension } \\
\hline Yes & $308(61.6)$ \\
\hline No & $184(36.8)$ \\
\hline Missing & $8(1.6)$ \\
\hline \multicolumn{2}{|l|}{ Congestive heart failure } \\
\hline Yes & $35(7.0)$ \\
\hline No & $435(87.0)$ \\
\hline Missing & $30(6.0)$ \\
\hline \multicolumn{2}{|l|}{ Dyspnea } \\
\hline Yes & $7(1.4)$ \\
\hline
\end{tabular}

Table 1 (continued)

\begin{tabular}{ll}
\hline Variable & Total, $n(\%)$ \\
\hline No & $464(92.8)$ \\
Missing & $29(5.8)$ \\
Smoking history & \\
Yes & $53(10.6)$ \\
No & $407(81.4)$ \\
Missing & $40(8.0)$ \\
Severe COPD & \\
Yes & $21(4.2)$ \\
No & $451(90.2)$ \\
Missing & $28(5.6)$ \\
Dialysis & \\
Yes & $2(0.4)$ \\
No & $469(93.8)$ \\
Missing & $29(5.8)$ \\
Acute renal failure & \\
Yes & $7(1.4)$ \\
No & $459(91.8)$ \\
Missing & $34(6.8)$ \\
\hline
\end{tabular}

$A S A$, American Society of Anesthesiologist; $A A$, African American; $C O P D$, chronic obstructive pulmonary disease; $I Q R$, interquartile range

$67 \%$ of the time (serious complication, 0.670 ; $95 \% \mathrm{CI} 0.612-$ 0.730 and any complication, $0.671 ; 95 \%$ CI $0.609-0.731)$. Model discrimination was weakest for VTE (C-statistic 0.538, 95\% CI 0.378-0.698; Brier score, 0.077) and SSI (Cstatistic 0.566, 95\%CI 0.466-0.666; Brier score 0.069). Additionally, only $57.8 \%$ and $71.2 \%$ of patients who experienced readmission (C-statistic $0.578,95 \%$ CI $0.468-0.688$ ) or mortality (C-statistic $0.712,95 \% \mathrm{CI} 0.508-0.917$ ) were correctly identified by the model. The SRC model was best at predicting non-home discharge (C-statistic $0.781,95 \% \mathrm{CI}$ 0.718-0.844; Brier score 0.024), reoperation (C-statistic 0.775, 95\% CI 0.647-0.904; Brier score 0.023), UTI (C-statistic $0.764,95 \%$ CI $0.659-0.868$; Brier score 0.025$)$, and pneumonia (C-statistic 0.722, 95\%CI 0.598-0.845; Brier score 0.031). Receiver operating characteristic (ROC) curves were defined and depicted for each complication type (Fig. 2).

\section{Discussion}

As patient outcomes become increasingly linked to provider reimbursement, heightened emphasis has been placed on proper patient risk stratification, as well as on adequate informed consent. The Centers for Medicare and Medicaid services created the Quality Improvement Organization in 2014 as a means to improve the quality of care for Medicare beneficiaries with a goal to decrease surgical complications. ${ }^{25}$ Surgeons should therefore comprehensively assess surgical risk not only to inform patients of the expected perioperative course but also to identify modifiable risk factors in hopes of improving patient outcomes. Risk assessment or clinical prediction tools have been developed to guide shared decision-making and define 
Table 2 Postoperative outcomes

\begin{tabular}{lllll}
\hline Outcome & $\begin{array}{l}\text { All cases, } \\
n(\%)\end{array}$ & $\begin{array}{l}\text { Minor hepatectomy, } \\
n(\%)\end{array}$ & $\begin{array}{l}\text { Major hepatectomy, } \\
n(\%)\end{array}$ & $p$ \\
\hline Total & 500 & $324(64.8)$ & $176(35.2)$ & \\
Serious complications & $98(19.6)$ & $46(14.2)$ & $52(29.5)$ & $<0.001$ \\
Any complications & $107(21.4)$ & $50(15.4)$ & $57(32.4)$ & $<0.001$ \\
Pneumonia & $17(3.4)$ & $7(2.2)$ & $10(5.7)$ & 0.067 \\
Cardiac complication & $10(2.0)$ & $3(0.9)$ & $7(4.0)$ & 0.038 \\
Surgical site infection & $37(7.5)$ & $21(6.5)$ & $16(9.1)$ & 0.29 \\
Urinary tract infection & $13(2.6)$ & $5(1.6)$ & $8(4.6)$ & 0.073 \\
Venous thromboembolism & $16(3.2)$ & $9(2.8)$ & $10(4.0)$ & 0.60 \\
Renal failure & $22(4.4)$ & $12(3.7)$ & $19(11.2)$ & 0.36 \\
30-day readmission & $33(7.0)$ & $14(4.6)$ & $8(4.6)$ & 0.013 \\
Reoperation & $11(2.4)$ & $3(0.9)$ & $4(2.3)$ & 0.006 \\
30-day mortality & $7(1.4)$ & $3(0.9)$ & $7(4.4)$ & 0.250 \\
Non-home discharge & $11(2.5)$ & $4(1.4)$ & & 0.062 \\
\hline
\end{tabular}

benchmark values for surgical outcomes. ${ }^{26}$ Tools such as the ACS NSQIP SRC are typically derived using retrospective data on routinely collected pre- and intraoperative factors. Previous authors have evaluated the SRC's performance to predict outcomes after general surgery, emergency surgery, orthopedic surgery, and gynecological surgery and have noted heterogeneous results. ${ }^{27-29}$ For example, Mogal et al. reported that the SRC had a good ability to predict outcomes among patients undergoing pancreaticoduodenectomy. ${ }^{30}$ In contrast, Beal et al. noted that the actual proportion of patients experiencing a complication was much higher than the median-predicted risk for patients undergoing liver and pancreatic surgery. ${ }^{21}$ Therefore, the actual performance of SRC remains a topic of debate. In addition, the performance of the SRC has not been previously examined specifically among the elderly. As the risk of complications may be very different among the elderly than the general population, accurate preoperative risk assessment among the aged population is critical. The current study was important because it specifically sought to externally validate and examine the accuracy of the ACS NSQIP SRC to predict outcomes among elderly patients undergoing liver resection of HCC using a large, multi-institutional international database. Of note, the ACS NSQIP SRC failed to estimate accurately the risk of many adverse outcomes after hepatectomy among the elderly. Specifically, the incidence of renal failure and VTE was underestimated by the SRC, while the calculator overestimated the risk for 30-day readmission and non-home discharge. Furthermore, the prediction model performed no better than random chance at discriminating patients who experienced a SSI, VTE, readmission, and 30-day mortality.
Table 3 Observed incidence versus predicted risk for postoperative outcomes

\begin{tabular}{lll}
\hline Outcome & \multicolumn{2}{l}{ All cases $N=500$} \\
\cline { 2 - 3 } & Observed incidence \% & Predicted risk median \% (IQR) \\
\hline Serious complications & 19.6 & $15.5(11.2,21.9)$ \\
Any complications & 21.4 & $17.7(13.1,23.9)$ \\
Pneumonia & 3.4 & $3.3(1.7,4.6)$ \\
Cardiac complication & 2.0 & $1.3(0.7,2.6)$ \\
Surgical site infection & 7.5 & $6.8(5.1,9.1)$ \\
Urinary tract infection & 2.6 & $2.4(1.9,3.1)$ \\
Venous thromboembolism & 3.2 & $1.8(1.5,3.1)$ \\
Renal failure & 4.4 & $1.0(0.5,2.0)$ \\
30-day readmission & 7.0 & $9.4(7.4,12.8)$ \\
Reoperation & 2.4 & $2.2(1.7,3.4)$ \\
30-day mortality & 1.4 & $0.8(0.3,2.2)$ \\
Non-home discharge & 2.5 & $5.7(3.3,11.7)$ \\
\hline
\end{tabular}


Table 4 Discrimination (Cstatistics) of 12 outcomes

\begin{tabular}{lllll}
\hline & All cases & & & \\
\cline { 2 - 5 } Model & $\begin{array}{l}\text { C- } \\
\text { statistic }\end{array}$ & $95 \%$ CI & $p$ & Brier score \\
\hline Serious complications & 0.670 & $0.612-0.730$ & $<0.001$ & 0.149 \\
Any complications & 0.671 & $0.609-0.731$ & $<0.001$ & 0.159 \\
Pneumonia & 0.722 & $0.598-0.845$ & 0.002 & 0.031 \\
Cardiac complication & 0.705 & $0.533-0.877$ & 0.027 & 0.019 \\
Surgical site infection & 0.566 & $0.466-0.666$ & 0.182 & 0.069 \\
Urinary tract infection & 0.764 & $0.659-0.868$ & 0.001 & 0.025 \\
Venous thromboembolism & 0.538 & $0.378-0.698$ & 0.601 & 0.077 \\
Renal failure & 0.682 & $0.554-0.811$ & 0.004 & 0.077 \\
Readmission & 0.578 & $0.468-0.688$ & 0.138 & 0.064 \\
Reoperation & 0.775 & $0.647-0.904$ & 0.001 & 0.023 \\
Mortality & 0.712 & $0.508-0.917$ & 0.054 & 0.014 \\
Non-home discharge & 0.781 & $0.718-0.844$ & 0.001 & 0.024 \\
\hline
\end{tabular}
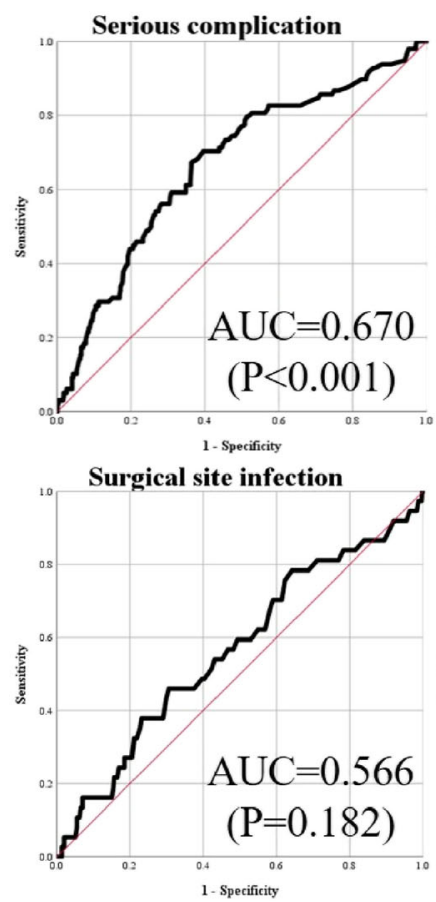

Readmission

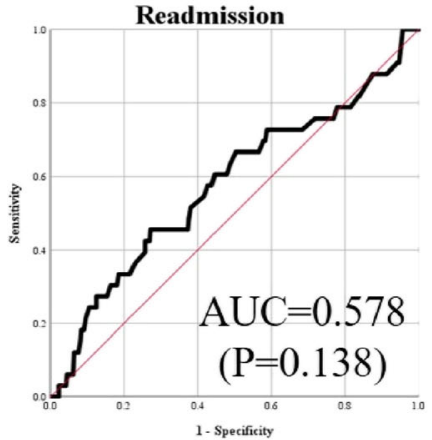

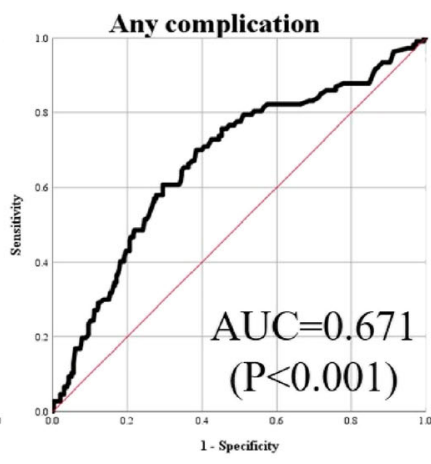

Urinary tract infection

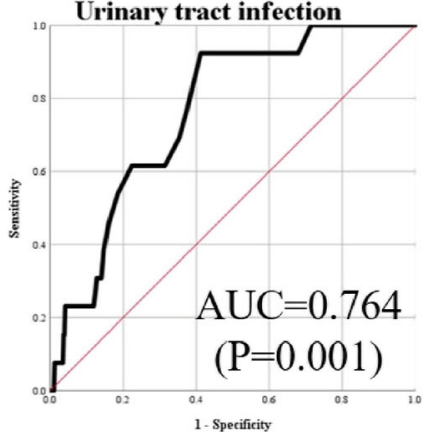

Reoperation

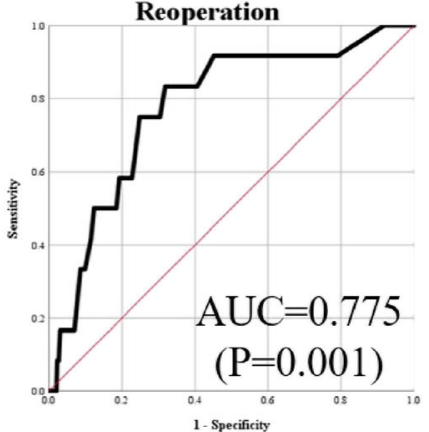

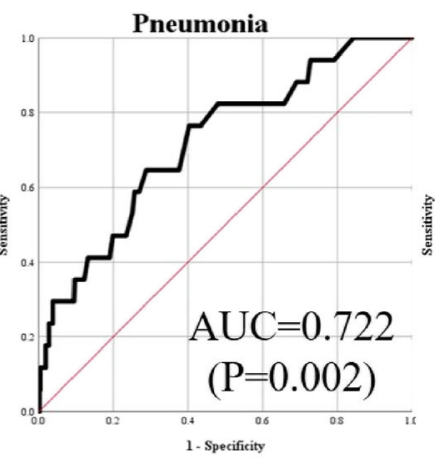
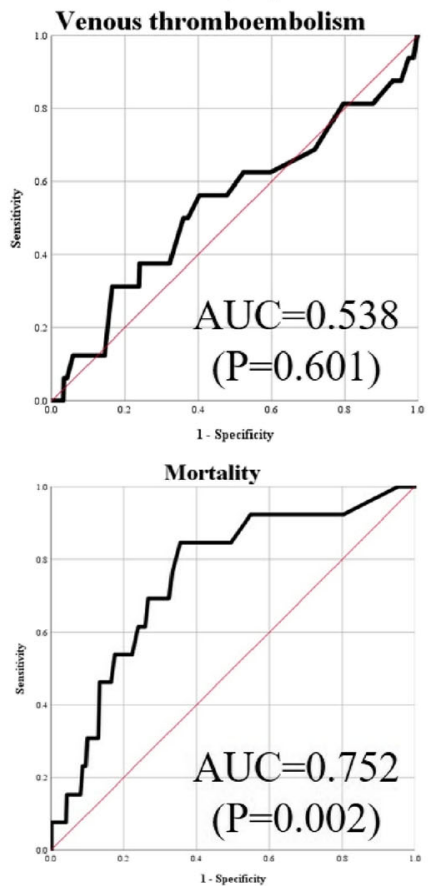

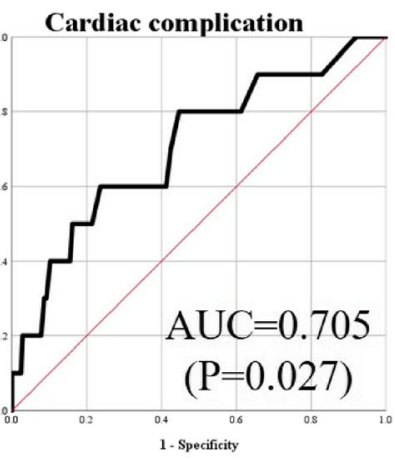

Renal failure
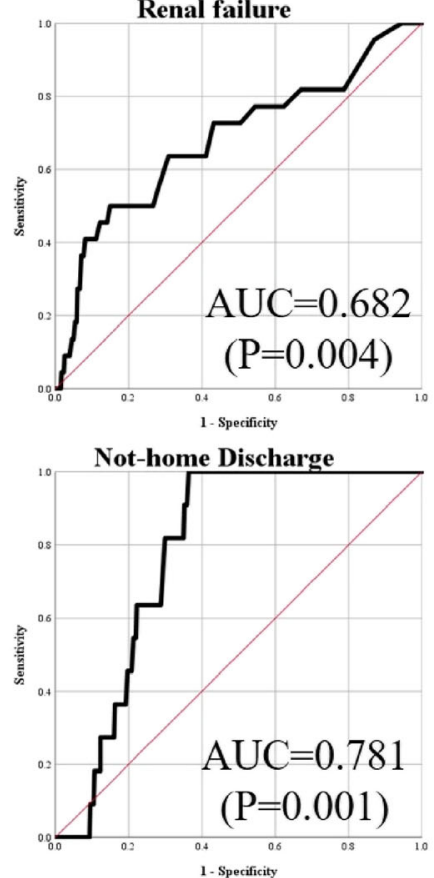

Fig. 2 Comparison of observed incidence of postoperative outcomes stratified by the risk categories of the risk calculator 
Together, the data suggest that the SRC was not a reliable tool to estimate the risk of perioperative complications among the elderly who were undergoing a hepatic resection for HCC.

Prognostication based on individual patient characteristics may be a more helpful way to inform patients of their specific peri- and postoperative risks. To this point, Kishida et al. noted that elderly patients undergoing hepatectomy for HCC had a greater likelihood of experiencing grade $3 \mathrm{a}$ or higher ClavienDindo complications compared with younger patients. ${ }^{31}$ In fact, the incidence of complications among the elderly has been estimated to be as high as $50 \%$, yet the range of risk can vary considerably. ${ }^{31-33}$ For patients undergoing liver resection, the most common complications include SSI, deepspace infection, UTI, bile leakage, and liver failure. ${ }^{34,35}$ The ASC NSQIP SRC provided estimates on the risk for some of the more common complications like SSI and UTI, yet data from the current study demonstrated that the model performed relatively poor. Specifically, among patients who experienced a SSI, the SRC had correctly assigned these patients a higher risk score only $56 \%$ of the time. Additionally, SRC routinely overestimated the incidence of non-home discharge and readmission. In fact, the accuracy of the SRC to predict readmission among elderly patients was only slightly better than chance (C-statistic, 0.578). Interestingly, Paredes et al. had previously noted that, while elderly patients did not have an increased risk of readmission compared with younger patients, elderly patients had higher rates of non-index readmission. ${ }^{36}$ In turn, the poor performance of the SRC to predict readmission may be due to the fact that the SRC was derived from NSQIP data, which were limited to the same hospital readmission within 30 days. Collectively, the data serve to highlight the limitations of the SRC to stratify elderly patients with regard to the risk of various complications - especially readmission-following resection of HCC.

While chronological age has been associated with outcomes following hepatic resection, physiological age may be more important in estimating the risk of postoperative outcomes. ${ }^{8,37,38}$ In particular, some groups have suggested that some measures of physiological fitness or frailty should be incorporated into tools to predict morbidity and mortality. ${ }^{32,36}$ To this end, the ACS NSQIP SRC accounted for the functional status of the patient at the time of operation with the "dependence" variable (i.e., independent, partially dependent, totally dependent). This variable may not have been adequate to capture the true physiological status of elderly patients and thereby have contributed to the general poor performance of the SRC. Rather, Saraiva and colleagues have reported that functional status defined according to metabolic equivalents (METs) was a more powerful means to assess the risk of surgical morbidity among elderly patients undergoing cancer surgery. ${ }^{39}$ Studies from our group and others have also suggested that frailty and sarcopenia may be indicative of a patient's physiologic age and consequentially serve as a better predictor of postoperative outcomes. ${ }^{40-42}$ To this point, Robinson et al. reported that geriatric assessment markers of frailty, disability, and medical comorbidity were strongly associated with 6-month postoperative mortality and postdischarge institutionalization following a major operation among the elderly. ${ }^{43}$ While the SRC incorporated a number of measures, the inability to better account for physiologic age, frailty, and exercise tolerance among the elderly limited the accuracy and applicability of the tool.

While the SRC tool did not provide an accurate means to estimate the risk of morbidity among elderly patients undergoing hepatic resection for HCC, such tools may be important to help guide practitioner and patient-level decision-making. ${ }^{44}$ The identification of patients at high risk of morbidity may allow for preemptive intervention. For example, Landefeld et al. reported that the implementation of a program consisting of patient-centered care that emphasized independence/mobility, pro-active discharge planning, and intensive review of predischarge medications improved the ability of older patients to be discharged at home rather than to long-term care institutions. ${ }^{45}$ In a separate study, Carli and colleagues suggested that a prehabilitation home-base program consisting of strength and endurance exercises, as well as improvements in nutritional status, could optimize elderly patients prior to surgical intervention. ${ }^{46}$ Future risk estimation tools should aim to better identify elderly patients at highest risk of postoperative complications, non-home discharge, and readmission to target these individuals for preoperative interventions such as prehabilitation.

The current study had several limitations that need to be considered when interpreting the data. Similar to other retrospective cohort studies, the data was subject to information bias. While the ACS NSQIP data was collected by professional coders that had undergone training to ensure the accuracy of the documentation, data in the current study was collected by clinicians. To limit possible information bias, definitions of the variables based on ACS NSQIP were provided to ensure adequate categorization. Though the multi-institutional nature of the analytic cohort allowed for a "real-word" attempt at validating the SRC using a diverse population, there exists a possibility of institution practices affecting the results. In order to limit this, most patients were recruited from major referral centers given that the overwhelming majority of liver resections for $\mathrm{HCC}$ among the elderly are performed at tertiary centers. ${ }^{47}$ Another limitation of the SRC itself was the lack of specific factors related to liver function that may better predict postoperative course (MELD score, INR, presence of cirrhosis, etc.).

In conclusion, the current work provides important insight regarding the use of the ACS NSQIP surgical risk calculator among elderly patients undergoing hepatic resection for HCC. Overall, the SRC performed poorly in this patient population. Specifically, the SRC underestimated the risk of certain 
complications such as VTE and renal failure, while providing no more than a "chance" estimation of other postoperative outcomes such as readmission. While the SRC performed best in estimating the risk of non-home discharge and mortality, the model failed to explain $20-30 \%$ of the patient-level variability. These data demonstrate that the ACS NSQIP SRC has little clinical application in risk stratifying elderly patients being considered for hepatic resection of HCC. Future refinement and development of better predictive tools to risk-stratify outcomes among elderly patients undergoing resection of HCC are warranted.

\section{Compliance with Ethical Standards}

The study was approved by the Institutional Review Boards of all participating institutions.

Conflict of Interest The authors declare that they have no conflict of interest.

\section{References}

1. Bray F, Ferlay J, Soerjomataram I, Siegel RL, Torre LA, Jemal A. Global cancer statistics 2018: GLOBOCAN estimates of incidence and mortality worldwide for 36 cancers in 185 countries. CA Cancer J Clin. 2018;68(6):394-424.

2. Bertuccio P, Turati F, Carioli G, Rodriguez T, La Vecchia C, Malvezzi M, et al. Global trends and predictions in hepatocellular carcinoma mortality. J Hepatol. 2017;67(2):302-9.

3. Beal EW, Tumin D, Kabir A, Moris D, Zhang XF, Chakedis J, et al. Trends in the Mortality of Hepatocellular Carcinoma in the United States. J Gastrointest Surg. 2017;21(12):2033-8.

4. White DL, Thrift AP, Kanwal F, Davila J, El-Serag HB. Incidence of Hepatocellular Carcinoma in All 50 United States, From 2000 Through 2012. Gastroenterology. 2017;152(4):812-20.e5.

5. Ueno M, Hayami S, Tani M, Kawai M, Hirono S, Yamaue H. Recent trends in hepatectomy for elderly patients with hepatocellular carcinoma. Surg Today. 2014;44(9):1651-9.

6. Rosero EB, Cheng GS, Khatri KP, Joshi GP. Evaluation of epidural analgesia for open major liver resection surgery from a US inpatient sample. Proceedings (Baylor University Medical Center). 2014;27(4):305-12.

7. Hankey BF, Ries LA, Kosary CL, Feuer EJ, Merrill RM, Clegg LX, et al. Partitioning linear trends in age-adjusted rates. Cancer causes \& control : CCC. 2000;11(1):31-5

8. Okinaga H, Yasunaga H, Hasegawa K, Fushimi K, Kokudo N. Short-Term Outcomes following Hepatectomy in Elderly Patients with Hepatocellular Carcinoma: An Analysis of 10,805 Septuagenarians and 2,381 Octo- and Nonagenarians in Japan. Liver Cancer. 2018;7(1):55-64.

9. Al-Refaie WB, Parsons HM, Henderson WG, Jensen EH, Tuttle TM, Vickers SM, et al. Major cancer surgery in the elderly: results from the American College of Surgeons National Surgical Quality Improvement Program. Annals of surgery. 2010;251(2):311-8.

10. Kneuertz PJ, Pitt HA, Bilimoria KY, Smiley JP, Cohen ME, Ko CY, et al. Risk of morbidity and mortality following hepato-pancreatobiliary surgery. Journal of gastrointestinal surgery : official journal of the Society for Surgery of the Alimentary Tract. 2012;16(9): 1727-35.

11. Oliveira-Cunha M, Malde DJ, Aldouri A, Morris-Stiff G, Menon $\mathrm{KV}$, Smith AM. Results of pancreatic surgery in the elderly: is age a barrier? HPB : the official journal of the International Hepato Pancreato Biliary Association. 2013;15(1):24-30.

12. Tzeng CW, Cooper AB, Vauthey JN, Curley SA, Aloia TA. Predictors of morbidity and mortality after hepatectomy in elderly patients: analysis of 7621 NSQIP patients. HPB : the official journal of the International Hepato Pancreato Biliary Association. 2014;16(5):459-68.

13. Bergquist JR, Shubert CR, Ubl DS, Thiels CA, Kendrick ML, Truty MJ, et al. Risk by indication for pancreaticoduodenectomy in patients 80 years and older: a study from the American College of Surgeons National Surgical Quality Improvement Program. HPB : the official journal of the International Hepato Pancreato Biliary Association. 2016;18(11):900-7.

14. Cho SW, Steel J, Tsung A, Marsh JW, Geller DA, Gamblin TC. Safety of Liver Resection in the Elderly: How Important Is Age? Annals of surgical oncology. 2011;18(4):1088-95.

15. Ruzzenente A, Conci S, Ciangherotti A, Campagnaro T, Valdegamberi A, Bertuzzo F, et al. Impact of age on short-term outcomes of liver surgery: Lessons learned in 10-years' experience in a tertiary referral hepato-pancreato-biliary center. Medicine. 2017;96(20):e6955.

16. Merkow RP, Bilimoria KY, Tomlinson JS, Paruch JL, Fleming JB, Talamonti MS, et al. Postoperative Complications Reduce Adjuvant Chemotherapy Use in Resectable Pancreatic Cancer. Annals of surgery. 2014;260(2):372-7.

17. Wu W, He J, Cameron JL, Makary M, Soares K, Ahuja N, et al. The Impact of Postoperative Complications on the Administration of Adjuvant Therapy Following Pancreaticoduodenectomy for Adenocarcinoma. Annals of surgical oncology. 2014;21(9):287381 .

18. Grady D, Berkowitz SA. Why is a good clinical prediction rule so hard to find? Archives of Internal Medicine. 2011;171(19):1701-2.

19. Wilson PW, D'Agostino RB, Levy D, Belanger AM, Silbershatz H, Kannel WB. Prediction of coronary heart disease using risk factor categories. Circulation. 1998;97(18):1837-47.

20. Cologne KG, Keller DS, Liwanag L, Devaraj B, Senagore AJ. Use of the American College of Surgeons NSQIP Surgical Risk Calculator for Laparoscopic Colectomy: how good is it and how can we improve it? Journal of the American College of Surgeons. 2015;220(3):281-6.

21. Beal EW, Lyon E, Kearney J, Wei L, Ethun CG, Black SM, et al. Evaluating the American College of Surgeons National Surgical Quality Improvement project risk calculator: results from the U.S Extrahepatic Biliary Malignancy Consortium. HPB : the official journal of the International Hepato Pancreato Biliary Association. 2017;19(12):1104-11.

22. Pencina MJ, D'Agostino RB. Evaluating Discrimination of Risk Prediction Models: The C Statistic. JAMA. 2015;314(10):1063-4.

23. Austin PC, Steyerberg EW. Interpreting the concordance statistic of a logistic regression model: relation to the variance and odds ratio of a continuous explanatory variable. BMC Med Res Methodol. 2012;12:82.

24. Rufibach K. Use of Brier score to assess binary predictions. J Clin Epidemiol. 2010;63(8):938-9; author reply 9.

25. Centers for Medicare and Medicaid Services. The Administration, Cost, and Impact of the Quality Improvement Organization Program for Medicare Beneficiaries for Fiscal Year 20172018. Available from: https://www.cms.gov/Medicare/QualityInitiatives - Patient-Assessment-Instruments/ QualityImprovementOrgs/Downloads/Annual-Report-toCongress-QIO-Program-Fiscal-Year-2017.pdf. Accessed 4 Jan 2019. 
26. Rix TE, Bates T. Pre-operative risk scores for the prediction of outcome in elderly people who require emergency surgery. World Journal of Emergency Surgery. 2007;2(1):16.

27. Edelstein AI, Kwasny MJ, Suleiman LI, Khakhkhar RH, Moore MA, Beal MD, et al. Can the American College of Surgeons Risk Calculator Predict 30-Day Complications After Knee and Hip Arthroplasty? The Journal of arthroplasty. 2015;30(9 Suppl):5-10.

28. Adegboyega TO, Borgert AJ, Lambert PJ, Jarman BT. Applying the National Surgical Quality Improvement Program risk calculator to patients undergoing colorectal surgery: theory vs reality. American journal of surgery. 2017;213(1):30-5.

29. Teoh D, Halloway RN, Heim J, Vogel RI, Rivard C. Evaluation of the American College of Surgeons National Surgical Quality Improvement Program Surgical Risk Calculator in Gynecologic Oncology Patients Undergoing Minimally Invasive Surgery. Journal of minimally invasive gynecology. 2017;24(1):48-54.

30. Mogal HD, Fino N, Clark C, Shen P. Comparison of observed to predicted outcomes using the ACS NSQIP risk calculator in patients undergoing pancreaticoduodenectomy. Journal of surgical oncology. 2016;114(2):157-62.

31. Kishida N, Hibi T, Itano O, Okabayashi K, Shinoda M, Kitago M, et al. Validation of hepatectomy for elderly patients with hepatocellular carcinoma. Ann Surg Oncol. 2015;22(9):3094-101.

32. Joseph B, Zangbar B, Pandit V, Fain M, Mohler MJ, Kulvatunyou N, et al. Emergency General Surgery in the Elderly: Too Old or Too Frail? J Am Coll Surg. 2016;222(5):805-13.

33. Vitale A, Spolverato G, Bagante F, Gani F, Popescu I, Marques HP, et al. A multi-institutional analysis of elderly patients undergoing a liver resection for intrahepatic cholangiocarcinoma. J Surg Oncol. 2016;113(4):420-6.

34. Jin S, Fu Q, Wuyun G, Wuyun T. Management of posthepatectomy complications. World J Gastroenterol. 2013;19(44): 7983-91.

35. Lafaro K, Buettner S, Maqsood H, Wagner D, Bagante F, Spolverato G, et al. Defining Post Hepatectomy Liver Insufficiency: Where do We stand? J Gastrointest Surg. 2015;19(11):2079-92.

36. Paredes AZ, Beal EW, Bagante F, Dillhoff ME, Cloyd JM, Pawlik TM. Patterns of readmission among the elderly after hepatopancreatobiliary surgery. American journal of surgery. 2018.

37. Adam R, Frilling A, Elias D, Laurent C, Ramos E, Capussotti L, et al. Liver resection of colorectal metastases in elderly patients. The British journal of surgery. 2010;97(3):366-76.
38. Yasunaga H, Horiguchi H, Matsuda S, Fushimi K, Hashimoto H, Ohe K, et al. Relationship between hospital volume and operative mortality for liver resection: Data from the Japanese Diagnosis Procedure Combination database. Hepatology research : the official journal of the Japan Society of Hepatology. 2012;42(11):1073-80.

39. Saraiva MD, Karnakis T, Gil-Junior LA, Oliveira JC, Suemoto CK, Jacob-Filho W. Functional Status is a Predictor of Postoperative Complications After Cancer Surgery in the Very Old. Ann Surg Oncol. 2017;24(5):1159-64.

40. Wagner D, DeMarco MM, Amini N, Buttner S, Segev D, Gani F, et al. Role of frailty and sarcopenia in predicting outcomes among patients undergoing gastrointestinal surgery. World J Gastrointest Surg. 2016;8(1):27-40.

41. Gani F, Cerullo M, Amini N, Buettner S, Margonis GA, Sasaki K, et al. Frailty as a Risk Predictor of Morbidity and Mortality Following Liver Surgery. J Gastrointest Surg. 2017;21(5):822-30.

42. Valero V, Amini N, Spolverato G, Weiss MJ, Hirose K, Dagher NN, et al. Sarcopenia adversely impacts postoperative complications following resection or transplantation in patients with primary liver tumors. J Gastrointest Surg. 2015;19(2):272-81.

43. Robinson TN, Eiseman B, Wallace JI, Church SD, McFann KK, Pfister SM, et al. Redefining geriatric preoperative assessment using frailty, disability and co-morbidity. Ann Surg. 2009;250(3):449-55.

44. Cohen ME, Liu Y, Ko CY, Hall BL. An Examination of American College of Surgeons NSQIP Surgical Risk Calculator Accuracy. J Am Coll Surg. 2017;224(5):787-95.e1.

45. Landefeld CS, Palmer RM, Kresevic DM, Fortinsky RH, Kowal J. A randomized trial of care in a hospital medical unit especially designed to improve the functional outcomes of acutely ill older patients. N Engl J Med. 1995;332(20):1338-44.

46. Carli F, Brown R, Kennepohl S. Prehabilitation to enhance postoperative recovery for an octogenarian following robotic-assisted hysterectomy with endometrial cancer. Can J Anaesth. 2012;59(8): 779-84.

47. Cerullo M, Chen SY, Dillhoff M, Schmidt C, Canner JK, Pawlik TM. Association of Hospital Market Concentration With Costs of Complex Hepatopancreaticobiliary Surgery. JAMA surgery. 2017;152(9):e172158.

Publisher's Note Springer Nature remains neutral with regard to jurisdictional claims in published maps and institutional affiliations. 